\title{
NEARLY COMPACT AND CONTINUOUS NORMAL FORM GAMES: CHARACTERIZATIONS AND EQUILIBRIUM EXISTENCE
}

\author{
C. J. HARRIS, M. B. STINCHCOMBE, AND W. ZAME
}

\begin{abstract}
Normal form games are nearly compact and continuous (NCC) if they can be understood as games played on strategy spaces that are dense subsets of the strategy spaces of larger compact games with jointly continuous payoffs. There are intrinsic algebraic, measure theoretic, functional analysis, and finite approximability characterizations of NCC games. NCC games have finitely additive equilibria, and all their finitely additive equilibria are equivalent to countably additive equilibria on metric compactifications. The equilibrium set of an NCC game depends upper hemicontinuously on the specification of the game and contains only the limits of approximate equilibria of approximate games.
\end{abstract}

\section{INTRODUCTION}

This paper answer the questions, "What is there that is intrinsic in a game $\Gamma$, specified only by the player set, $I$, the strategy sets, $S_{i}$, and the bounded utility functions, $u_{i}: S \rightarrow \mathbb{R}, S=\times_{i \in I} S_{i}$, that leads to equilibrium existence, upperhemicontinuity of the equilibrium correspondence, and continuity of the approximate equilibrium correspondence?" and "What topological and measure theoretic structures are entailed?"

When each $S_{i}$ is finite, Nash [11] defined the now standard equilibrium concept for non-cooperative game theory and showed that equilibria in mixed strategies exist. To generalize Nash's existence result to games with infinite strategy spaces, Fan [6] and Glicksberg [8] make two inter-related choices: for finiteness they substitute jointly continuous utilities on compact metric strategy spaces; for mixed strategies, they employ the dual of the space of continuous functions, the countably additive Borel probabilities. These choices lead to three properties critical to the interpretations of game theoretic models: existence, upper hemicontinuity of the equilibrium correspondence, and continuity of the approximate equilibrium correspondence.

Date: May 31,2001. 
Existence guarantees that the models make predictions. Upper hemicontinuity of the equilibrium set guarantees that small misspecification of the utilities or the strategy sets of a game do not lead to equilibria that are too far from the true set of equilibria. This is crucial to the Fan and Glicksberg existence proofs which proceed by approximating the compact strategy spaces by sequences of large finite sets. Fudenberg and Levine [7], prove that the approximate equilibrium-approximate strategy set correspondence is continuous, thereby providing a clean interpretation of games with infinite sets of strategies.

A game $\Gamma=\left(S_{i}, u_{i}\right)_{i \in I}$ is nearly compact and continuous (NCC) if each strategy set $S_{i}$ can be imbedded as a dense subset of a compact $\widehat{S}_{i}$ in such a fashion that each $u_{j}$ can be extended to a jointly continuous function on $\times_{i \in I} \widehat{S}_{i}$. Any such larger game, $\widehat{\Gamma}=\left(\widehat{S}_{i}, \hat{u}_{i}\right)_{i \in I}$, is called a called a continuous compact imbedding, or cci, of $\Gamma$. Normal form games that fail to be NCC include the normal forms of most non-trivial extensive form games. These other normal form games are treated in the companion piece, Stinchcombe [14], which develops the requisite theory of integration for non-measurable functions.

The following are the essential results for NCC games.

(1) The equivalence of five intrinsic characterizations of games:

(a) nearly compact and continuous - the game can be understood as one being played on dense subsets of the strategy spaces of a compact and continuous game;

(b) algebraic - the uniform approximability of the utility functions by polynomials in extended sections;

(c) measure theoretic - the integrability of utility functions with respect to all products of finitely additive mixed strategies; ${ }^{1}$

(d) functional analysis - sup norm precompactness of the sections of the utility functions;

(e) finite approximability - the strategy sets of the players can be uniformly approximated in the "most difference it can make to anyone" metric.

(2) If a game has one cci, then it has many. The maximal and minimal cci's can be characterized, and all cci's of a given game are equivalent in the following senses:

(a) their equilibrium sets are non-empty and payoff equivalent;

(b) the equilibrium set of a smaller cci is the set of restrictions of equilibrium strategies in any larger cci;

\footnotetext{
${ }^{1}$ The failure of the utility function to be integrable is what necessitates the development of a theory of integration for non-measurable function in non-NCC games in [14].
} 
(c) the equilibrium set of a larger cci is the set of extensions of equilibrium strategies in any smaller cci.

(3) The play of finitely additive mixed strategies corresponds to a particular class of cci's, a class that contains the minimal and the maximal cci's.

(4) Identifying equivalent strategies leads to the minimal cci, which is metric. From this, existence, upper hemicontinuity and continuity follow.

The next section contains the results, the following contains examples demonstrating the reach and limitations of the results, the last concludes.

\section{Results}

This section begins with the definitions and equivalence of five conditions: (a) $\Gamma=$ $\left(S_{i}, u_{i}\right)_{i \in I}$ being NCC, (b) each $u_{i}$ being nearly polynomial in the extended sections of the $u_{k}, k \in I,(\mathrm{c})$, the utility functions being integrable against all products of finitely additive strategies, (d) the sections of the utility functions being precompact, and (e) the strategy sets of the players can be uniformly approximated in the "most difference it can make to anyone" metric. An examination of the various compact imbeddings follows. The metrizability of the minimal compact imbedding leads to equilibrium existence, the upper hemicontinuity of the equilibrium correspondence, and the continuity of the approximate equilibrium correspondence. A treatment of finitely additive equilibria end the section.

\subsection{Definitions and Equivalence.}

2.1.1. Nearly compact and continuous games. Let $X$ be a non-empty set. A compact imbedding of $X$ is a pair $((\widehat{X}, \widehat{\tau}), \varphi)$ where $(\widehat{X}, \widehat{\tau})$ is a compact Hausdorff space (cHs), and $\varphi: X \rightarrow \widehat{X}$ imbeds $X$ in $\widehat{X}$ so that $\varphi(X)$ is $\widehat{\tau}$-dense in $\widehat{X}$. Note that $\varphi$ may be many-to-one.

For any $\mathrm{cHs} \widehat{X}, C(\widehat{X})$ denotes the set of continuous function on $\widehat{X}$. Let $f$ be a bounded, real-valued function on $X$. A compact imbedding $((\widehat{X}, \widehat{\tau}), \varphi)$ continuously extends $f$ if there exists an $\hat{f} \in C(\widehat{X})$ such that for all $x \in X$, $\hat{f}(\varphi(x))=f(x)$. A necessary condition for a compact imbedding to extend $f$ is that $\varphi$ must separate any points separated by $f$.

Let $F$ be a set of bounded, real-valued functions on $X$. An $F$-imbedding of $X$ is a compact that continuously extends every $f \in F$. The denseness of $\varphi(X)$ in $\widehat{X}$ has two implications: first, $\hat{f}$ is unique if it exists; second, if $\hat{f}$ exists, then $\sup _{x \in X}|f(x)|=\max _{\hat{x} \in \hat{X}}|\hat{f}(x)| . F$-imbeddings exist for every $F$, and any compact imbedding is homeomorphic to an $F$-imbedding for some $F$, details and references are below in $\S 2.2 .1$. 
Definition 1. A game $\widehat{\Gamma}=\left(\widehat{S}_{i}, \hat{u}_{i}\right)_{i \in I}$ is a continuous compact imbedding (cci) of $\Gamma=\left(S_{i}, u_{i}\right)_{i \in I}$ if for each $i \in I$ there exists a compact Hausdorff topology $\widehat{\tau}_{i}$ for $\widehat{S}_{i}$ and a function $\varphi_{i}: S_{i} \rightarrow \widehat{S}_{i}$ such that

(1) each $\left(\left(\widehat{S}_{i}, \widehat{\tau}_{i}\right), \varphi_{i}\right)$ is a compact imbedding of $S_{i}, i \in I$, and

(2) $((\widehat{S}, \widehat{\tau}), \varphi)$ is a $\left\{u_{1}, \ldots, u_{I}\right\}$-imbedding of $S$ where $\widehat{S}:=\times_{i \in I} \widehat{S}_{i}, \widehat{\tau}$ is the product topology on $\widehat{S}, \varphi\left(s_{1}, \ldots, s_{I}\right):=\left(\varphi_{1}\left(s_{1}\right), \ldots, \varphi_{I}\left(s_{I}\right)\right)$, and the $\hat{u}_{i}$ are the continuous extensions of the $u_{i}$.

A crucial aspect of this definition is that the $\hat{u}_{i}$ must be jointly continuous on $\widehat{S}$.

Definition 2. A game $\Gamma$ is nearly compact and continuous (NCC) if it has a continuous compact imbedding.

Intuitively, a game $\Gamma$ is NCC if it can be understood as a game played on dense subsets of the strategy spaces of a compact and continuous game.

2.1.2. Nearly polynomial in extended sections games. For $s \in S$ and $t_{i} \in S_{i}$, the two game theoretic notations $s \backslash t_{i}$ and $\left(t_{i}, s_{-i}\right)$ will be used interchangeably as dictated by convenience.

If $f_{i}$ is a function on $S_{i}$, then its product extension, $f_{i}^{e}$, is the function on $S$ defined by $f_{i}^{e}\left(s_{i}, s_{-i}\right)=f_{i}\left(s_{i}\right)$ for all $\left(s_{i}, s_{-i}\right) \in S$. $F_{i}^{e}$ denotes the set of product extensions of a class $F_{i}$ of function on $S_{i}$. Given a game $\Gamma=\left(S_{i}, u_{i}\right)_{i \in I}, i$ 's utility sections is the class of functions $\mathcal{U}_{i}=\left\{s_{i} \mapsto u_{k}\left(s \backslash s_{i}\right): k \in I, s \in S\right\}$. A crucial implication of $\widehat{\Gamma}$ being a cci of $\Gamma$ is that each $\left(\left(\widehat{S}_{i}, \widehat{\tau}_{i}\right), \varphi_{i}\right)$ is a $\mathcal{U}_{i}$-imbedding of $S_{i}$.

Of particular interest will be polynomials in the set of utility section product extensions, $\mathcal{U}_{i}^{e}$. Given a class $H$ of functions on $S, \mathcal{P}(H)$ denotes the set of polynomials in $H$, that is, the set of finite linear combinations of functions of the form

$$
g(s)=\left(h_{1}(s)\right)^{\alpha_{1}} \cdot\left(h_{2}(s)\right)^{\alpha_{2}} \cdot \cdots \cdot\left(h_{M}(s)\right)^{\alpha_{M}}
$$

where $M \in \mathbb{N}$ and $\alpha_{m} \in\{0\} \cup \mathbb{N}, m=1, \ldots, M$.

Definition 3. A game $\Gamma$ is nearly polynomial in its extended sections (NPES) if each $u_{j}, j \in I$, is in the sup norm closure of the polynomials in the utility section product extensions, that $i s, u_{j} \in \operatorname{cl} \mathcal{P}\left(\left\{\mathcal{U}_{i}^{e}: i \in I\right\}\right)$.

Intuitively, a game is NPES if information about the sections of the utility functions is enough to reconstruct the whole utility function using only polynomial operations. 
2.1.3. Integrable games. Let $\mathcal{X}$ denote a field (not necessarily a $\sigma$-field) of subsets of a set $X$. A simple, real-valued $\mathcal{X}$-measurable function on $X$ is a finite linear combination of indicator functions of sets in $\mathcal{X}{ }^{2} B(\mathcal{X})$ denotes the set of uniform limits of simple, real-valued, $\mathcal{X}$-measurable functions. Any $f \in B(\mathcal{X})$ is integrable against any finitely additive probability on $\mathcal{X}$.

If $\Sigma_{i}$ is a field of subsets of $S_{i}$ for each $i \in I$, then $\times_{i \in I} \Sigma_{i}$ denotes the product field generated by the $\Sigma_{i}$, that is, the smallest field containing all sets of the form $\times_{i \in I} E_{i}, E_{i} \in \Sigma_{i} . B\left(\times_{i \in I} \Sigma_{i}\right)$ is the set of functions that can be integrated against any product of finitely additive probabilities on the $\Sigma_{i}$ 's. $2^{S_{i}}$ denotes the set of all subsets of $S_{i}$.

Definition 4. A game $\Gamma$ is integrable if each $u_{j}, j \in I$, belongs to $B\left(\times_{i \in I} 2^{S_{i}}\right)$.

Intuitively, the integrable games are the ones for which an expected utility can be assigned to independent play of any profile of finitely additive strategies.

2.1.4. Utility precompact games. Let $d$ be a metric on a space $X$. A set $T \subset X$ is $d$-precompact if for every $\epsilon>0$, there exists a finite $\epsilon$-net, that is, a finite set $T_{\epsilon}$ such that for all $t \in T, d\left(t, T_{\epsilon}\right)<\epsilon$. The sup norm metric on the set of bounded, real-valued functions on $S_{i}$ is denoted $\rho_{i}$.

Definition 5. A game $\Gamma$ is utility precompact (UPC) if for all $i \in I, \mathcal{U}_{i}$ is $\rho_{i}$-precompact.

Intuitively, a game is UPC if its sets of utility sections are not too large.

2.1.5. Finitely approximable games. For any uniformly bounded set of functions $F$ on a set $X$, and any $x, y \in X$, define the pseudo-metric $d_{F}(x, y)$ by $d_{F}(x, y)=$ $\sup _{f \in F}|f(x)-f(y)|$. The following is an interesting reformulation of the pseudometrics $d_{\mathcal{U}_{i}}(\cdot, \cdot)$ on the $S_{i}$.

$$
d_{\mathcal{U}_{i}}\left(s_{i}, t_{i}\right)=\sup _{w_{i} \in \mathcal{U}_{i}}\left|w_{i}\left(s_{i}\right)-w_{i}\left(t_{i}\right)\right|=\max _{k \in I} \sup _{s \in S}\left|u_{k}\left(s \backslash s_{i}\right)-u_{k}\left(s \backslash t_{i}\right)\right| .
$$

This is Fudenberg and Levine's [7] "most utility difference it can make to anyone" pseudo-metric. Note that $d_{\mathcal{U}_{i}}\left(s_{i}, t_{i}\right)=0$ if and only if $s_{i}$ and $t_{i}$ are strategically equivalent. $d_{\mathcal{U}_{i}}$ is a metric on equivalence classes of strategies.

Definition 6. A game $\Gamma$ is finitely approximable (FA) if for all $i \in I, S_{i}$ is $d_{\mathcal{U}_{i}}$-precompact.

\footnotetext{
${ }^{2}$ Specifically, if it is a function of the form $f(x)=\sum_{m=1}^{M} r_{m} 1_{E_{m}}(x)$ where $M \in \mathbb{N}, r_{m} \in \mathbb{R}$, $E_{m} \in \mathcal{X}$, and $1_{E_{m}}(x)$ is the function equal to 1 if $x \in E_{m}$ and equal to 0 otherwise.
} 


\subsubsection{Equivalence. It is now time to prove}

Theorem 1. The following five conditions are equivalent:

(a) $\Gamma$ is $N C C$.

(b) $\Gamma$ is NPES.

(c) $\Gamma$ is integrable.

(d) $\Gamma$ is $U P C$.

(e) $\Gamma$ is $F A$.

Proof: The structure of the proof is $\mathrm{NCC} \Rightarrow \mathrm{NPES} \Rightarrow$ integrable $\Rightarrow \mathrm{UPC} \Rightarrow \mathrm{NCC}$, and $\mathrm{UPC} \Leftrightarrow \mathrm{FA}$.

NCC $\Rightarrow$ NPES: Suppose that $\left(\widehat{S}_{i}, \hat{u}_{i}\right)_{i \in I}$ is a cci of $\Gamma$. For each $i \in I$, let $\varphi_{i}$ denote the imbedding of $S_{i}$ in $\widehat{S}_{i}$. By definition, each $\hat{u}_{k} \in C(\widehat{S})$. We must show that each $u_{k} \in \operatorname{cl} \mathcal{P}\left(\left\{\mathcal{U}_{i}^{e}: i \in I\right\}\right)$. Because $\varphi(S)$ is dense in each $\widehat{S}$, and because each $\mathcal{U}_{i}$ contains the sections of all of the $u_{k}$, proving the following Lemma will complete the proof.

Lemma 1. If $(X, \tau)$ is the product of cHs's $\left(X_{i}, \tau_{i}\right)$ with the product topology, and if $f \in C(X)$, then $f \in \operatorname{cl} \mathcal{P}\left(\left\{\mathcal{S}_{i}^{e}: i \in I\right\}\right)$ where $\mathcal{S}_{i}=\left\{x_{i} \mapsto f\left(x \backslash x_{i}\right): x \in X\right\}$.

Proof: For each $i$, let $\tau_{i}^{\circ} \subset \tau_{i}$ denote the weakest topology on $X_{i}$ making each $f_{i} \in \mathcal{S}_{i}$ continuous. Define $x_{i} \sim_{i} y_{i}$ if for all $G_{i} \in \tau_{i}^{\circ}, x_{i} \in G_{i}$ if and only if $y_{i} \in G_{i}$. Denote by $X_{i}^{\prime}$ the quotient space of $X_{i}$ divided by $\sim_{i}$. Denote by $\tau_{i}^{\prime}$ the identification topology on $X_{i}^{\prime}$, so that each $\left(X_{i}^{\prime}, \tau_{i}^{\prime}\right)$ is a cHs. Denote by $p_{i}$ the projection of $X_{i}$ onto $X_{i}^{\prime}$ (i.e. $p_{i}\left(x_{i}\right)$ is the equivalence class of $x_{i}$ ). Denote by $p$ the corresponding projection of $X$ onto $X^{\prime}=\times_{i \in I} X_{i}^{\prime}$ with the product topology.

It is immediate that $f p^{-1}$ is single-valued. Since $f \in C(X)$, this is sufficient condition for $\hat{f}:=f p^{-1} \in C\left(X^{\prime}\right)[4$, Theorem VI.4.2(3), p. 125]. Since the collection of functions $\left\{C^{e}\left(X_{i}^{\prime}\right): i \in I\right\}$ separate points in $X^{\prime}$, the Stone-Weierstrass theorem implies that $C\left(X^{\prime}\right)=\operatorname{cl} \mathcal{P}\left(\left\{C^{e}\left(X_{i}^{\prime}\right): i \in I\right\}\right)$.

Each $g_{i} \in \mathcal{S}_{i}$ belongs to $C\left(X_{i}\right)$ and $g_{i} p_{i}^{-1}$ is single-valued so that $\hat{g}_{i}:=g_{i} p_{i}^{-1}$ belongs to $C\left(X_{i}^{\prime}\right)$. By construction, the collection $\widehat{\mathcal{S}}_{i}=\left\{\hat{g}_{i}: g_{i} \in \mathcal{S}_{i}\right\}$ separates points in $X_{i}^{\prime}$. Therefore, by the Stone-Weierstrass theorem, $C\left(X_{i}^{\prime}\right)=\operatorname{cl} \mathcal{P}\left(\widehat{\mathcal{S}}_{i}\right)$. Since polynomials in polynomials are again polynomials, this implies that $\hat{f} \in \operatorname{cl} \mathcal{P}\left(\left\{\widehat{\mathcal{S}}_{i}^{e}: i \in I\right\}\right)$, which directly implies that $f \in \operatorname{cl} \mathcal{P}\left(\left\{\mathcal{S}_{i}^{e}: i \in I\right\}\right)$.

NPES $\Rightarrow$ integrable: Suppose that $\Gamma$ is NPES. Pick arbitrary $\epsilon>0$ and $j \in I$. We will show that there exists a simple, $\times_{i \in I} 2^{S_{i}}$-measurable function, $v_{j}^{\epsilon}$, such that $\sup _{s \in S}\left|u_{j}(s)-v_{j}^{\epsilon}(s)\right|<\epsilon$. 
Let $[a, b]$ be a compact interval such that for all $k \in I$ and all $s \in S, a \leq u_{k}(s) \leq b$. Because $\Gamma$ is NPES, there exists a $w_{j}^{\epsilon}$ in $\mathcal{P}\left(\left\{\mathcal{U}_{i}^{e}: i \in I\right\}\right)$ such that $\sup _{s \in S} \mid u_{j}(s)-$ $w_{j}^{\epsilon}(s) \mid<\epsilon / 2$. The function $w_{j}^{\epsilon}$ is a polynomial in $M$ elements of $\left\{\mathcal{U}_{i}^{e}: i \in I\right\}$ for some $M \in \mathbb{N}$. Thus, there are $M$ functions, $h_{1}, \ldots, h_{M}$ in $\left\{\mathcal{U}_{i}^{e}: i \in I\right\}$ and a polynomial, $p_{j}^{\epsilon}:[a, b]^{M} \rightarrow \mathbb{R}$, such that for all $s \in S$,

$$
w_{j}^{\epsilon}(s)=p_{j}^{\epsilon}\left(h_{1}(s), \ldots, h_{M}(s)\right) .
$$

Being continuous, $p_{j}^{\epsilon}$ is uniformly continuous on the compact set $[a, b]^{M}$. Therefore it is possible to pick a $\delta>0$ such that for all $x, y \in[a, b]^{M}$, if $\left|x_{m}-y_{m}\right|<\delta$, $m=1, \ldots, M$, then $\left|p_{j}^{\epsilon}(x)-p_{j}^{\epsilon}(y)\right|<\epsilon / 2$.

For each $m, h_{m}$ is of the form $h_{m}(s)=f_{j_{m}}^{e}\left(s_{j_{m}}, s_{-j_{m}}\right)$ for some $j_{m} \in I$ and

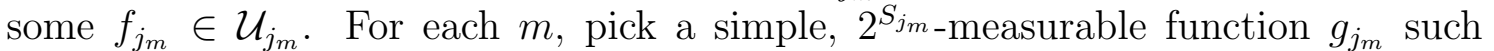
that $\sup _{s_{j_{m}} \in S_{j_{m}}}\left|f_{j_{m}}\left(s_{j_{m}}\right)-g_{j_{m}}\left(s_{j_{m}}\right)\right|<\delta$. Define $h_{m}^{\delta}(s)=g_{j_{m}}^{e}\left(s_{j_{m}}, s_{-j_{m}}\right)$ so that $\sup _{s \in S}\left|h_{m}(s)-h_{m}^{\delta}(s)\right|<\delta$. Each $h_{m}^{\delta}$ is a simple, $\times_{i \in I} 2^{S_{i}}$-measurable function. Because the class of simple, $\times_{i \in I} 2^{S_{i}}$-measurable functions is an algebra, the function

$$
v_{j}^{\epsilon}(s)=p_{j}^{\epsilon}\left(h_{1}^{\delta}(s), \ldots, h_{M}^{\delta}(s)\right)
$$

is also a simple, $\times_{i \in I} 2^{S_{i}}$-measurable function. Further, $\sup _{s \in S}\left|w_{j}^{\epsilon}(s)-v_{j}^{\epsilon}(s)\right|<\epsilon / 2$.

By the triangle inequality,

$$
\sup _{s \in S}\left|u_{j}(s)-v_{j}^{\epsilon}(s)\right| \leq \sup _{s \in S}\left(\left|u_{j}(s)-w_{j}^{\epsilon}(s)\right|+\left|w_{j}^{\epsilon}(s)-v_{j}^{\epsilon}(s)\right|\right) .
$$

This last term is in turn less than or equal to

$$
\sup _{s \in S}\left|u_{j}(s)-w_{j}^{\epsilon}(s)\right|+\sup _{s \in S}\left|w_{j}^{\epsilon}(s)-v_{j}^{\epsilon}(s)\right|,
$$

and each of these last two terms is less than $\epsilon / 2$.

Integrable $\Rightarrow$ UPC: Suppose that $\Gamma$ is integrable. Pick an arbitrary $\epsilon>0$ and $i \in I$. We will show that there is a finite set $T_{i}^{\epsilon}$ of bounded functions on $S_{i}$ such that for all $f_{i} \in \mathcal{U}_{i}, \rho_{i}\left(f_{i}, T_{i}^{\epsilon}\right)<\epsilon$.

Because $\Gamma$ is integrable, for each $k \in I$, there exists a simple $\times_{j \in I} 2^{S_{j}}$-measurable function function $v_{k}^{\epsilon}$ such that

$$
\sup _{s \in S}\left|u_{k}(s)-v_{k}^{\epsilon}(s)\right|<\epsilon .
$$

Since $v_{k}^{\epsilon}$ is a simple, product field measurable function, it has only finitely many distinct sections. Therefore, there is a finite collection, $F_{k}^{\epsilon}=\left\{f_{k, 1}^{\epsilon}, \ldots, f_{k, M_{k}}^{\epsilon}\right\}$, such that $\left\{s_{i} \mapsto v_{k}^{\epsilon}\left(s \backslash s_{i}\right): s \in S\right\} \subset F_{k}^{\epsilon}$.

To complete the proof, set $T_{i}^{\epsilon}=\cup_{k \in I} F_{k}^{\epsilon}$. 
UPC $\Rightarrow$ NCC: Suppose that $\Gamma$ is not NCC. We will show that $\Gamma$ is not UPC.

For each $i \in I$, let $\left(\left(\widehat{S}_{i}, \widehat{\tau}_{i}\right), \varphi_{i}\right)$ be a $\mathcal{U}_{i}$-imbedding of $S_{i}$. For each $j \in I$, the function $\hat{u}_{j}$ is well-defined on $\varphi(S)$ by $\hat{u}_{j}(\hat{s})=u_{j}\left(\varphi^{-1}(\hat{s})\right.$. Since $\Gamma$ is not NCC, at least one $k \in I, \hat{u}_{k}$ does not have a jointly continuous extension from $\varphi(S)$ to $\widehat{S}$. Because $\varphi(S)$ is dense in $\widehat{S}$, there exist $\epsilon>0$ and two nets $s^{\alpha}$ and $t^{\beta}$ in $\varphi(S)$ converging to some $\hat{s}^{\circ} \in \widehat{S}$, such that $\liminf _{\alpha} u_{k}\left(\varphi^{-1}\left(s^{\alpha}\right)\right) \geq \lim \sup _{\beta} u_{k}\left(\varphi^{-1}\left(t^{\beta}\right)\right)+2 \epsilon$. Because $I$ is finite, this means that for at least one $j \in I$, the mappings $s_{j} \mapsto u_{k}\left(\varphi^{-1}\left(s^{\alpha}\right) \backslash s_{j}\right)$ and $s_{j} \mapsto u_{k}\left(\varphi^{-1}\left(t^{\beta}\right) \backslash s_{j}\right)$ in $\mathcal{U}_{j}$ are infinitely often at sup norm distance at least $\epsilon$ from each other. This means that $\mathcal{U}_{j}$ is not $\rho_{j}$-precompact.

UPC $\Leftrightarrow$ FA: Suppose first that $\Gamma$ is UPC and pick an arbitrary $\epsilon>0$. Let $T_{i}^{\epsilon}$ be an $\epsilon$-net for $\mathcal{U}_{I}$ in the $\rho_{i}$ metric. For all $s_{i}, t_{i} \in S_{i},\left|d_{T_{i}^{\epsilon}}\left(s_{i}, t_{i}\right)-d_{\mathcal{U}_{i}}\left(s_{i}, t_{i}\right)\right|<\epsilon$. Therefore proof that $\Gamma$ is $\mathrm{FA}$ will be complete if we show the existence of a finite set $A_{i}^{\epsilon}$ such that $d_{T_{i}^{\epsilon}}\left(A_{i}^{\epsilon}, S_{i}\right)<\epsilon$.

Pick $a<b$ in $\mathbb{R}$ such that for all $w_{i} \in \mathcal{U}_{i}$ and all $s_{i} \in S_{i}, a \leq w_{i}\left(s_{i}\right) \leq b$. For integer $k \leq(b-a) / \epsilon$, let $R_{k}$ be the half-open interval $[a+(k-1) \epsilon, a+k \epsilon)$. Let $\mathcal{P}_{i, \epsilon}$ be the finite partition of $S_{i}$ generated by finite cover $\left\{f_{i}^{-1}\left(R_{k}\right): f_{i} \in T_{i}^{\epsilon}, k \leq(b-a) / \epsilon\right\}$. For each $E_{i} \in \mathcal{P}_{i, \epsilon}$, pick a point $a_{i, E_{i}} \in E_{i}$ and let $A_{i}^{\epsilon}$ be the finite set $\left\{a_{i, E_{i}}: E_{i} \in \mathcal{P}_{i, \epsilon}\right\}$. For all $s_{i} \in S_{i}$, there exists an $a_{i} \in A_{i}^{\epsilon}$ such that for all $f_{i} \in T_{i}^{\epsilon},\left|f_{i}\left(a_{i}\right)-f_{i}\left(s_{i}\right)\right|<\epsilon$, that is, $d_{T_{i}^{\epsilon}}\left(a_{i}, s_{i}\right)<\epsilon$.

Suppose now that $\Gamma$ is FA and pick an arbitrary $\epsilon>0$. Let $A_{i}$ be a finite $\epsilon$-net for $S_{i}$ in the $d_{\mathcal{U}_{i}}$ metric. The proof that $\Gamma$ is UPC will be complete if we show that there exists a finite $T_{i}^{\epsilon} \subset \mathcal{U}_{i}$ such that $\rho_{i}\left(T_{i}^{\epsilon}, \mathcal{U}_{i}\right)<2 \cdot \epsilon$.

For each $w_{i} \in \mathcal{U}_{i}$, define $x=x\left(w_{i}\right)$ as the point in $\mathbb{R}^{A_{i}}$ having $a_{i}$ 'th component equal to $w_{i}\left(a_{i}\right), a_{i} \in A_{i}$. Let $E$ be the set $x\left(\mathcal{U}_{i}\right)$. Since cl $E$ is compact, there is a finite $T_{i}^{\epsilon} \subset \mathcal{U}_{i}$ such that $x\left(T_{i}^{\epsilon}\right)$ is an $\epsilon$-net for $E$ in the metric generated by the norm $\|y\|=\max _{a_{i} \in A_{i}}\left|y_{a_{i}}\right|$. By the definition of $d_{\mathcal{U}_{i}}$, for all $s_{i} \in S_{i}$, there exists an $a_{i} \in A_{i}$ such that for all $w_{i} \in \mathcal{U}_{i},\left|w_{i}\left(a_{i}\right)-w_{i}\left(s_{i}\right)\right|<\epsilon$. Therefore, for all $w_{i} \in \mathcal{U}_{i}$, there is an $f_{i} \in T_{i}^{\epsilon}$ such that $\rho_{i}\left(f_{i}, w_{i}\right)<2 \cdot \epsilon$.

\subsection{On the Continuous Compact Imbeddings of a Game.}

2.2.1. Basics of continuous compact imbeddings. Let $X$ be a non-empty set and $F$ a class of bounded, real-valued functions on $X$. The following sketches the construction of the canonical $F$-imbedding, $\left(\left(\widehat{X}_{F}, \widehat{\tau}_{F}\right), \varphi_{F}\right)$.

(1) for each $f \in F$, let $\left[a_{f}, b_{f}\right]$ be a compact interval satisfying $a_{f} \leq f(X) \leq b_{f}$,

(2) imbed each $x \in X$ as $\varphi_{F}(x) \in \bar{X}:=\times_{f \in F}\left[a_{f}, b_{f}\right]$ where $\varphi_{F}(x)$ as the vector defined by $\operatorname{proj}_{f} \varphi_{F}(x)=f(x)$,

(3) give $\bar{X}$ the compact product topology, and 
(4) define $\widehat{X}_{F}$ as the closure of $\varphi_{F}(X)$ in $\bar{X}$ and let $\widehat{\tau}_{F}$ denote the relative topology on $\widehat{X}_{F} \cdot{ }^{3}$

$\varphi_{F}(x) \neq \varphi_{F}(y)$ in the canonical $F$-imbedding if and only if $F$ separates $x, y \in X$. Since the product topology is defined by the continuity of the projection mappings, $\left(\left(\widehat{X}_{F}, \widehat{\tau}_{F}\right), \varphi_{F}\right)$ is indeed an $F$-imbedding of $X$. Since projections separate points in $\widehat{X}_{F}$, the Stone-Weierstrass theorem implies that the sup norm closed algebra, $C\left(\widehat{X}_{F}\right)$, is the closure of the algebra $\mathcal{P}\left(\left\{\operatorname{proj}_{f}: f \in F\right\}\right)$. This means that every function in $\operatorname{cl} \mathcal{P}(F)$ has a continuous extension to $\widehat{X}_{F}$.

Let $B_{X}$ denote the set of bounded functions on $X$. Define a partial order on subsets of $B_{X}$ by $F \succeq G$ iff $\operatorname{cl} \mathcal{P}(F) \supset \operatorname{cl} \mathcal{P}(G)$. Let $\left(\left(\widehat{X}_{F}, \widehat{\tau}_{F}\right), \varphi_{F}\right)$ be the canonical $F$-imbedding of $X$, and let $\left(\left(\widehat{X}_{G}, \widehat{\tau}_{G}\right), \varphi_{G}\right)$ be the canonical $G$-imbedding. The next results follow from [4, Theorems XI.8.2 and XIII.6.5, pp. 243 and 289]:

(1) If $F \succeq G$, then there is a continuous surjection, $\psi: \widehat{X}_{F} \rightarrow \widehat{X}_{G}$ such that for all $x \in X, \psi\left(\varphi_{F}(x)\right)=\varphi_{G}(x)$;

(2) if $F \sim G$, then $\psi$ is a homeomorphism, and if $F \succ G$, then $\psi$ is many-to-one;

(3) if $((\widehat{X}, \widehat{\tau}), \varphi)$ is a compact imbedding of $X$, then it is homeomorphic to the canonical $F$-imbedding for some $F$ - for each $g \in C(\widehat{X})$, define a bounded function on $X$ by $f_{g}(x)=g(\varphi(x))$, and set $F=\left\{f_{g}: g \in C(\widehat{X})\right\}$.

These last three results imply that for any $F \subset B_{X}$, there is a maximal $F$ imbedding, namely the $B_{X}$-imbedding, and a minimal one, homeomorphic to the $\mathcal{P}(F)$-imbedding. The last point implies that the partial ordering on $B_{X}$ can identified with a partial ordering on spaces homeomorphic to the canonical compact imbeddings.

2.2.2. The maximal, minimal, and intermediate cci's. Fix a game $\Gamma=\left(S_{i}, u_{i}\right)_{i \in I}$ and partially order the compact imbeddings, $\left(\left(\widehat{S}_{i}, \widehat{\tau}_{i}\right), \varphi_{i}\right)$ of $S_{i}$ by $\succeq_{i}$ as above. If each $u_{k}$ is jointly continuous on $\widehat{S}$, then for each $i \in I$, each section $f_{i} \in \mathcal{U}_{i}$ must have a continuous extension to $\widehat{S}_{i}$. In other words, if $\left(\widehat{S}_{i}, \hat{u}_{i}\right)_{i \in I}$ is a cci of $\left(S_{i}, u_{i}\right)_{i \in I}$, then each $\widehat{S}_{i}$ must be at least as large as the $\mathcal{U}_{i}$-imbedding. All such imbeddings give rise to a cci when $\Gamma$ is NCC. Let $\left(\left(\bar{S}_{i}, \bar{\tau}_{i}\right), \bar{\varphi}_{i}\right)$ denote the canonical $\mathcal{U}_{i}$-imbedding.

\footnotetext{
${ }^{3}$ Some examples: Taking $F$ to be the set of continuous functions on $X=[0, \infty)$ having limits as $x \uparrow \infty$ means that $\left(\widehat{X}_{F}, \widehat{\tau}_{F}\right)$ is homeomorphic to the classical 1-point compactification of $[0, \infty)$; Taking $F$ to be the set of bounded, continuous functions on a completely regular topological space $(X, \tau)$ means that $\left(\widehat{X}_{F}, \widehat{\tau}_{F}\right)$ is homeomorphic to the Stone-Čech compactification of $(X, \tau)$; Taking $F$ to be the bounded measurable functions on a measure space $(X, \mathcal{X})$ gives the Stone space for $L^{\infty}$. Compact imbeddings are, essentially, compactifications of $X$ that do not involve a topology on the space $X$ and may be many-to-one.
} 
Theorem 2. If $\Gamma$ is NCC and for all $i \in I\left(\left(\widehat{S}_{i}, \widehat{\tau}_{i}\right), \varphi_{i}\right)$ is an imbedding between the $\mathcal{U}_{i}$-imbedding and the $B_{S_{i}}$-imbedding, then for all $i \in I$, there exists a $\hat{u}_{i} \in C(\widehat{S})$ such that $\left(\widehat{S}_{i}, \hat{u}_{i}\right)_{i \in I}$ is a cci of $\Gamma$.

Proof: Suppose first that for all $i \in I,\left(\left(\widehat{S}_{i}, \widehat{\tau}_{i}\right), \varphi_{i}\right) \sim_{i}\left(\left(\bar{S}_{i}, \bar{\tau}_{i}\right), \bar{\varphi}_{i}\right)$. Because $\Gamma$ is NPES, each $u_{k}$ is the uniform limit of a sequence $u_{k}^{n}$ of functions in $\mathcal{P}\left(\left\{\mathcal{U}_{i}^{e}: i \in I\right\}\right)$. Because polynomials in continuous functions are continuous and each $f_{i} \in \mathcal{U}_{i}$ has a continuous extension from $\varphi_{i}\left(S_{i}\right)$ to $S_{i}$, each $u_{k}^{n}$ has a continuous extension, $\hat{u}_{k}^{n}$ from $\varphi(S)$ to $S$. Since the sup norm distance between any pair $u_{k}^{n}$ and $u_{k}^{n^{\prime}}$ is the same as the sup norm distance between $\hat{u}_{k}^{n}$ and $\hat{u}_{k}^{n^{\prime}}$, the sequence $\hat{u}_{k}^{n}$ is Cauchy, hence has a limit $\bar{u}_{k} \in C(\widehat{S})$. The function $\bar{u}_{k}$ extends $u_{k}$ from $\varphi(S)$ to $S$. This means that $\left(\widehat{S}_{i}, \bar{u}_{i}\right)_{i \in I}$ is a cci of $\Gamma$.

Now suppose that $\left(\left(\widehat{S}_{i}, \widehat{\tau}_{i}\right), \varphi_{i}\right) \succeq_{i}\left(\left(\bar{S}_{i}, \bar{\tau}_{i}\right), \bar{\varphi}_{i}\right)$. Let $\psi_{i}$ denote the continuous surjection of $\widehat{S}_{i}$ onto $\bar{S}_{i}$ such that for all $s_{i} \in S_{i}, \psi_{i}\left(\varphi_{i}\left(s_{i}\right)\right)=\bar{\varphi}_{i}\left(s_{i}\right)$. Define $\psi(\hat{s})=$ $\left(\psi_{1}\left(\hat{s}_{1}\right), \ldots, \psi_{I}\left(\hat{s}_{I}\right)\right)$. For each $k \in I$, define $\hat{u}_{k}$ by $\hat{u}_{k}(\hat{s})=\bar{u}_{k}(\psi(\hat{s}))$. Being the composition of continuous functions, $\hat{u}_{k}$ is continuous. Further, $\hat{u}_{k}$ extends $u_{k}$ so that $\left(\widehat{S}_{i}, \hat{u}_{i}\right)_{i \in I}$ is a cci of $\Gamma$.

Recall that $\mathrm{f}$ or any uniformly bounded set of functions $F$ on a set $X$, and any $x, y \in X$, the pseudo-metric $d_{F}(x, y)$ is defined by $d_{F}(x, y)=\sup _{f \in F}|f(x)-f(y)|$. The following implies that the cHs's, $\left(\bar{S}_{i}, \bar{\tau}_{i}\right)$, in the minimal cci's can be metrized by $d_{\mathcal{U}_{i}}(\cdot, \cdot)$. In words, NCC games require only metrizable cci's, and the "most utility difference it makes to anyone" metric is the relevant one.

Lemma 2. Suppose that $(X, \tau)$ is a cHs. $\tau$ is metrizable if and only if there exists a sup norm compact $F \subset C(X)$ that separates points. Further, for any such $F, \tau$ can be metrized by $d_{F}(\cdot, \cdot)$.

Proof: Suppose that $\tau$ is metrized by the metric $d$. For each $x^{n}$ in a countable dense subset of $X$, define $f^{n}(x)=d\left(x, x^{n}\right) / n$. The collection $\left\{f^{n}: n \in \mathbb{N}\right\}$ separates points, and because $f^{n} \rightarrow 0$ in the sup norm, it is sup norm precompact.

Suppose that $F$ separates points and is sup norm precompact. Because $(X, \tau)$ is a cHs, it is normal (i.e. $T_{4}$ ), and for any normal space, a net $x^{\alpha} \rightarrow_{\tau} x$ iff $\forall f \in C(X)$, $f\left(x^{\alpha}\right) \rightarrow f(x)$. Since $F$ separates points, $C(X)=\operatorname{cl} \mathcal{P}(F)$ by the Stone-Weierstrass theorem. Thus, $x^{\alpha} \rightarrow_{\tau} x$ iff $\forall f \in \mathrm{cl} F, f\left(x^{\alpha}\right) \rightarrow f(x)$. Define $g^{\alpha}(\cdot)$ and $g(\cdot)$ on cl $F$ by $g^{\alpha}(f)=f\left(x^{\alpha}\right)$ and $g(f)=f(x)$. Rewriting the previous "iff" statement gives $x^{\alpha} \rightarrow_{\tau} x$ iff $g^{\alpha}$ converges pointwise to $g$. Since $g^{\alpha}, g \in C(\operatorname{cl} F)$ and $\operatorname{cl} F$ is compact, pointwise convergence of the $g^{\alpha}$ to $g$ is equivalent to uniform convergence. 
Rewriting once again, $x^{\alpha} \rightarrow_{\tau} x$ iff $\bar{d}_{F}\left(x^{\alpha}, x\right):=\max _{f \in \mathrm{cl} F}\left|f\left(x^{\alpha}\right)-f(x)\right| \rightarrow 0$. Note that $\bar{d}_{F}=d_{F}$.

2.2.3. Implications of metrizability. The next result shows that, after identifying the strategically equivalent strategies of an NCC game and adding some $d_{\mathcal{U}_{i}}$-limit points, what is left is a Fan-Glicksberg game. In the presence of jointly continuous payoffs, the generality gained in moving from compact metric to compact Hausdorff spaces of actions is illusory - it disappears after equivalent strategies are identified. This implies that Fan [6] and Glicksberg [8] achieved as much topological generality as possible.

Corollary 3. If for each $S_{i}$ there is a topology $\tau_{i}$ such that $\left(S_{i}, \tau_{i}\right)$ is a cHs and each $u_{i}$ is jointly continuous on $S$, then for each $i \in I, \bar{\varphi}_{i}\left(s_{i}\right)$ is a continuous surjection of $S_{i}$ onto $\bar{S}_{i}$, and $\bar{\varphi}_{i}\left(s_{i}\right)=\bar{\varphi}_{i}\left(t_{i}\right)$ if and only if $s_{i}$ and $t_{i}$ are strategically equivalent.

Proof: Taking $\iota_{i}$ as the identity mapping makes $\left(\left(S_{i}, \tau_{i}\right), \iota_{i}\right)$ a compact imbedding leading to a cci. Since $\left(\left(S_{i}, \tau_{i}\right), \iota_{i}\right)$ is a $\mathcal{U}_{i}$-imbedding, the rest follows.

If $\psi$ is a continuous surjection of a $\mathrm{cHs}\left(X^{\prime}, \tau^{\prime}\right)$ onto a compact metric space $(X, \tau)$, and $\mu$ is a countably additive probability on the Bore $\sigma$-field generated by $\tau$, then $\psi^{-1}(\mu):=\left\{\nu:(\forall G \in \tau)\left[\nu\left(\psi^{-1}(G)\right)=\mu(G)\right]\right\}$. If $E$ is a weak ${ }^{*}$ closed set of Borel probabilities on $\sigma(\tau)$, then $\psi^{-1}(E)$ is weak* closed. Recall that if $\left(\left(\widehat{S}_{i}, \widehat{\tau}_{i}\right), \varphi_{i}\right) \succeq_{i}\left(\left(\bar{S}_{i}, \bar{\tau}_{i}\right), \bar{\varphi}_{i}\right)$, then there is a continuous surjection $\psi_{i}: \widehat{S}_{i} \rightarrow \bar{S}_{i}$, and for $\hat{s} \in \widehat{S}, \psi(s):=\left(\psi_{1}\left(\hat{s}_{1}\right), \ldots, \psi_{I}\left(\hat{s}_{I}\right)\right)$.

Corollary 4. If $\Gamma$ is NCC and $\left(\widehat{S}_{i}, \hat{u}_{i}\right)_{i \in I}$ is a cci of $\Gamma$, then the closed, non-empty equilibrium set for $\widehat{\Gamma}$ is $\psi^{-1}(\bar{E})$ where $\bar{E}$ is the non-empty, closed set of equilibria for $\left(\bar{S}_{i}, \bar{u}_{i}\right)_{i \in I}$.

Proof: $\bar{E}$ is non-empty and closed by the proofs in [6] or [8]. Since the utilities are constant on $\psi^{-1}(\bar{s})$ for any $\bar{s} \in \bar{S}$, for any profile of strategies $\left(\nu_{i}\right)_{i \in I}$ in $\widehat{\Gamma}$, $\int_{\widehat{S}} \hat{u}_{k} d\left(\nu_{i}\right) i \in I=\int_{\bar{S}} \bar{u}_{k} d\left(\psi_{i}\left(\nu_{i}\right)\right) i \in I$. Thus, $\left(\nu_{i}\right)_{i \in I}$ is an equilibrium profile in $\widehat{\Gamma}$ if and only if $\left(\psi_{i}\left(\nu_{i}\right)\right) i \in I$ is an equilibrium profile in $\bar{\Gamma}$.

The last result show the strong sense in which cci's larger than $\left(\bar{S}_{i}, \bar{\varphi}_{i}\right)$ are redundantly large for NCC games. The only significant difference between the minimal and a larger cci is that the larger cci may contain more representations of strategically equivalent points. This conclusion fails rather dramatically for games that fail to be NCC - no compact imbeddings contain enough points, though larger spaces from nonstandard analysis do, see [14].

For any NCC game, the metrizability of each compact $\bar{\tau}_{i}$ implies there are sequences, $A_{i}^{n}$, of finite subsets of $S_{i}$, such that $d_{\mathcal{U}_{i}}\left(A_{i}^{n}, S_{i}\right) \rightarrow 0$, providing a second 
proof that all NCC games are finitely approximable. The next Corollary treats the upper hemicontinuity of the equilibrium correspondence. The routine proof is omitted.

Corollary 5. If $\widehat{\Gamma}=\left(\widehat{S}_{i}, \hat{u}_{i}\right)_{i \in I}$ is a cci for $\Gamma, d_{\mathcal{U}_{i}}\left(A_{i}^{n}, S_{i}\right) \rightarrow 0$, and $\left(\mu_{i}^{n}\right)_{i \in I}$ is a sequence of $\epsilon^{n}$-equilibria for the finite games $\left(A_{i}^{n}, u_{i}\right)_{i \in I}, \epsilon^{n} \geq 0, \epsilon^{n} \rightarrow 0$, then any accumulation point of $\psi^{-1}\left(\left(\mu_{i}^{n}\right)_{i \in I}\right)$ is an equilibrium for $\left(\widehat{S}_{i}, \hat{u}_{i}\right)_{i \in I}$.

The continuity of the approximate equilibrium correspondence follows directly from the corresponding result for Fan-Glicksberg games and the fact that each $\psi_{i}$ is an open mapping.

Corollary 6. If $\widehat{\Gamma}=\left(\widehat{S}_{i}, \hat{u}_{i}\right)_{i \in I}$ is a cci for $\Gamma$ and $\left(\nu_{i}\right)_{i \in I}$ is an equilibrium profile for $\widehat{\Gamma}$, then for any weak* open neighborhood $G$ of $\left(\nu_{i}\right)_{i \in I}$ and any $\epsilon>0$, there exists a finite game $\left(A_{i}, u_{i}\right)_{i \in I}$ such that $d_{\mathcal{U}_{i}}\left(A_{i}, S_{i}\right)<\epsilon$ and an $\epsilon$-equilibrium profile $\left(\mu_{i}\right)_{i \in I}$ for $\left(A_{i}, u_{i}\right)_{i \in I}$ such that $\psi^{-1}\left(\left(\mu_{i}\right)_{i \in I}\right) \cap G \neq \emptyset$.

2.2.4. Finitely additive equilibria. The cci approach compactifies before randomizing, and this leads to a compact set of countably additive mixed strategies. Directly compactifying the set of finitely supported mixed strategies leads to the weak* compact set of finitely additive strategies. Because the finitely supported strategies include probabilities $\mu$ such that for all measurable $E, \mu(E)=0$ or $\mu(E)=1$, this approach implicitly compactifies the pure strategies (see that finitely additive analysis of Example 1 below). Finitely additive randomization has two distinct roles in normal form games: first, it convexifies; second, it represents limits of approximate best responses. Because the implicit compactification is typically much larger than the minimal compactifications, there are many representations of equivalent strategies. Finitely additive game theory contains many redundant strategies.

A field $\Sigma_{i}$ of subsets of $S_{i}$ is adequate for $\Gamma$ if $\mathcal{U}_{i} \subset B\left(\Sigma_{i}\right)$. If $\Sigma_{i}$ is adequate, then any $B\left(\Sigma_{i}\right)$-imbedding must be a $\mathcal{U}_{i}$-imbedding. For a given adequate $\Sigma_{i}, \widehat{S}_{i}^{S t}=$ $\widehat{S}_{i}^{S t}\left(\Sigma_{i}\right)$ denotes the canonical $B\left(\Sigma_{i}\right)$-imbedding. It is known as the Stone space. To any finitely additive probability, $\mu_{i}$, on $\Sigma_{i}$, there corresponds a unique, countably additive, regular probability, $\mu_{i}^{S t}$, on the Borel $\sigma$-field of (any space homeomorphic to) $\widehat{S}_{i}^{S t}$. The probability $\mu_{i}^{S t}$ satisfies, for each $f_{i} \in B\left(\Sigma_{i}\right), \int f_{i} d \mu_{i}=\int \hat{f}_{i} d \mu_{i}^{S t}$ where $\hat{f}_{i}$ is the continuous extension of $f_{i}$.

Let $\Delta_{i}^{f a}=\Delta_{i}^{f a}\left(\Sigma_{i}\right)$ denote the finitely additive probabilities on $\Sigma_{i}$. If $\Gamma$ is NCC, then for all $k \in I$ and all profiles $\nu=\left(\nu_{i}\right)_{i \in I} \in \times_{i \in I} \Delta_{i}^{f a}, \int_{S} u_{k} d \nu$ is well-defined [5, Lemma III.2.16, p. 111]. Therefore, equilibria in finitely additive probabilities are well-defined. 
Theorem 3. If $\Gamma$ is NCC and $\left(\Sigma_{i}\right)_{i \in I}$ is an adequate collection, then the vector $\nu=\left(\nu_{i}\right)_{i \in I} \in \times_{i \in I} \Delta_{i}^{f a}$ is a finitely additive equilibrium for $\Gamma$ if and only if the vector $\nu^{S t}=\left(\nu_{i}^{S t}\right)_{i \in I}$ is an equilibrium for the (Stone) game $\widehat{\Gamma}^{S t}=\left(\widehat{S}_{i}^{S t}, \hat{u}_{i}^{S t}\right)_{i \in I}$. In particular, the set of finitely additive equilibria is weak* closed, non-empty, and payoff equivalent to the equilibria of any cci of $\Gamma^{4}$

\section{EXAMPLES}

NCC games fail to be compact metric space games with jointly continuous payoffs either by having strategy spaces with many representations of equivalent strategies, or by having strategy spaces that fail to contain limit points in the intrinsically defined pseudo-metric $d_{\mathcal{U}_{i}}$. The examples in this section demonstrate the reach and the limitation of the results leading to this conclusion.

First to be studied are single-player games, all of which are NCC. Some simple two player games follow. These show that being NCC rules out diagonal discontinuities. Special types of diagonal discontinuities were exploited by Dasgupta and Maskin [3] and Simon [12] in their studies of the equilibrium existence question for games with compact metric space $S_{i}$ 's and discontinuous payoffs. The last example in this section shows that $\Gamma$ being NCC rules out $\Gamma$ being the normal form of most extensive form games.

\subsection{Single Player Normal Form Games are NCC. The starting point is}

Lemma 7. If $I=\{1\}$, i.e. contains only one agent, then $\Gamma$ is $N C C$.

Proof: $\mathcal{U}_{1}=\left\{u_{1}\right\}$, and one point sets are precompact, so that $\Gamma$ is UPC.

Finitely additive and continuous compact imbedding analyses are informative.

3.1.1. The finitely additive analysis of single player games. Suppose that $\Sigma_{1}$ is adequate for the bounded utility function $u_{1}: S_{1} \rightarrow \mathbb{R}$, i.e. $\mathcal{U}_{1}=\left\{u_{1}\right\} \subset B\left(\Sigma_{1}\right)$. 1's equilibrium problem is to solve

$$
\max _{\nu_{1} \in \Delta_{1}^{f a}} \int_{S_{1}} u_{1}\left(s_{1}\right) d \nu_{1}\left(s_{1}\right) .
$$

Set $u_{1}^{*}=\sup u_{1}\left(S_{1}\right)$. To solve the problem it is sufficient to find a $\nu_{1}^{*}$ such that $\int_{S_{1}} u_{1}\left(s_{1}\right) d \nu_{1}^{*}\left(s_{1}\right)=u_{1}^{*}$. Because $\Sigma_{1}$ is adequate, for every $n \in \mathbb{N}$, there is a simple $\Sigma_{1}$-measurable function $u_{1}^{n}(\cdot)$ uniformly within $1 / n$ of $u_{1}(\cdot)$. Set $E_{n}=\left\{s_{1}: u_{1}^{n}\left(s_{1}\right) \geq\right.$

\footnotetext{
${ }^{4}$ While the first version of this paper was being written, Marinacci [9], now [10], independently showed that equilibria in finitely additive probabilities exist when $\Gamma$ is integrable.
} 
$\left.u_{1}^{*}-1 / n\right\}$ gives a sequence of sets in $\Sigma_{1}$ satisfying $E^{n+1} \cap E^{n} \neq \emptyset$ for all $n \in$ $\mathbb{N}$. If $\nu_{1}^{*}$ is any finitely additive probability assigning mass 1 to each $E^{n}$, then $\int_{S_{1}} u_{1}\left(s_{1}\right) d \nu_{1}^{*}\left(s_{1}\right)=u_{1}^{*}$.

The adequacy of $\Sigma_{1}$ means that finitely additive solutions to one person optimization problems exist. In many person games, the adequacy of $\Sigma_{i}$ means that for any $s \in S, i$ 's maximization problem,

$$
\max _{\nu_{i} \in \Delta_{i}^{f a}} \int_{S_{i}} u_{i}\left(s \backslash t_{i}\right) d \nu_{i}\left(t_{i}\right),
$$

always has a solution. Adequacy guarantees that there are finitely additive best responses to all pure strategies. Being NCC, equivalently, being integrable, means that this is sufficient for the existence of an equilibrium.

3.1.2. The cci analysis of single player games. In a single player game, $d_{\mathcal{U}_{1}}\left(s_{1}, t_{1}\right)=$ $\left|u_{1}\left(s_{1}\right)-u_{1}\left(t_{1}\right)\right|$. This is a pseudo-metric on $S_{1}$ that identifies indifferent strategies. Compactifying the pseudo-metric space $\left(S_{1}, d_{\mathcal{U}_{1}}\right)$ gives $\widehat{S}_{1}=\operatorname{cl} u_{1}\left(S_{1}\right) \subset \mathbb{R}$ with the usual topology, and gives $\hat{u}_{1}\left(\hat{s}_{1}\right)=\hat{s}_{1}$. Thus, $d_{\mathcal{U}_{1}}\left(A_{1}^{n}, S_{1}\right) \rightarrow 0$ if and only if $u_{1}\left(A_{1}^{n}\right) \rightarrow \operatorname{cl} u_{1}\left(S_{1}\right)$ in the usual Hausdorff sense. After compact imbedding, 1's equilibrium problem is (with $\Delta_{1}^{c a}$ being the countably additive Borel probabilities on $\widehat{S}_{1}$ )

$$
\max _{\nu_{1} \in \Delta_{1}^{c a}} \int_{\widehat{S}_{1}} \hat{u}_{1}\left(\hat{s}_{1}\right) d \nu_{1}\left(\hat{s}_{1}\right),
$$

and the solution, which is unique in these minimal cci's of single player games, is point mass on $u_{1}^{*} \in \widehat{S}_{1}$.

Being a $\mathcal{U}_{1}$-imbedding in a one person game means that solutions to optimization problems exist in the larger space $\widehat{S}_{1}$. In many person games, being a $\mathcal{U}_{i}$-imbedding means that for any $s \in S$, i's maximization problem,

$$
\max _{\hat{s}_{i} \in \widehat{S}_{i}} \hat{u}_{i}\left(\varphi(s) \backslash \hat{s}_{i}\right),
$$

always has a non-empty set of solutions, i.e. appropriate imbedding guarantees that there are best responses to all pure strategies. Being NCC means that this is sufficient for the existence of an equilibrium.

3.2. Some Two Person Normal Form Games. The first game is NCC, having only a 'vertical' discontinuity. The minimal cci adds just one $d_{\mathcal{U}_{i}}$-limit point at the discontinuity. The second game has a 'diagonal' discontinuity and is therefore not NCC though it does have a continuum of pure strategy equilibria. No compact imbedding will make the game continuous, and the expected utility associated with 
play of profiles of finitely additive strategies are not generally well-defined. The third game also has a diagonal discontinuity, and has no $\epsilon$ equilibria for any $\epsilon<1$.

3.2.1. A vertical discontinuity. The utilities $u_{i}\left(s_{i}, s_{j}\right)$ fail to be continuous at $s_{i}=1$ in the following $\mathrm{NCC}$ game.

Example 1. The game $\Gamma_{1}$ is specified by $I=\{1,2\}, S_{i}=[0,1]$, and the symmetric utility functions,

$$
u_{i}\left(s_{i}, s_{j}\right)=\left\{\begin{array}{cl}
s_{i}+s_{i} s_{j} & \text { if } s_{i}<1, \\
-1 & \text { if } s_{i}=1 .
\end{array}\right.
$$

Without imbedding (or something equivalent), this game has no equilibrium no matter what $j$ plays, $i$ can do better by moving closer to, but not quite reaching, $s_{i}=1$. Each $S_{i}=[0,1]$ can be viewed as a dense subset of the compact space $\widehat{S}_{i}=\left[0,1^{-}\right] \cup\{2\}$ after the imbedding

$$
\psi_{i}(r)= \begin{cases}r & \text { if } r<1, \\ 2 & \text { if } r=1 .\end{cases}
$$

Note that $\psi_{i}([0,1])=\left[0,1^{-}\right) \cup\{2\}$, a dense subset of $\widehat{S}_{i}$. Each $u_{i}(\cdot, \cdot)$ has a unique continuous extension, $\hat{u}_{i}$, from $\psi_{i}\left(S_{i}\right) \times \psi_{j}\left(S_{j}\right) \subset \widehat{S}_{i} \times \hat{S}_{j}$ to all of $\widehat{S}_{i} \times \hat{S}_{j}$. It is given by

$$
\hat{u}_{i}\left(\hat{s}_{i}, \hat{s}_{j}\right)=\left\{\begin{array}{cl}
\hat{s}_{i}+\hat{s}_{i} \hat{s}_{j} & \text { if } \hat{s}_{i} \leq 1^{-}, \\
-1 & \text { if } \hat{s}_{i}=2,
\end{array}\right.
$$

where, for numerical purposes, $1^{-}$is treated as 1 . The unique equilibrium is $\left(1^{-}, 1^{-}\right)$.

Finitely additive strategies that put mass 1 on all intervals of the form $(1-\epsilon, 1)$ are equilibria. Because finitely additive game theory corresponds to redundantly large cci's, there are many such finitely additive equilibria. They correspond to mixed strategies that put mass 1 on the strategic equivalents of $1^{-}$.

A final observation about this game is that sequences of finite approximations are $d_{\mathcal{U}_{i}}$-dense if and only if they Hausdorff approximate $[0,1)$ and eventually contain the point 1.

3.2.2. A diagonal discontinuity and many equilibria. The utilities $u_{i}\left(s_{i}, s_{j}\right)$ fail to be continuous at $s_{i}=s_{j}$ in the following non-NCC game.

Example 2. The game $\Gamma_{2}$ is specified by $I=\{1,2\}, S_{i}=[0,1]$, and the symmetric utility functions,

$$
u_{i}\left(s_{i}, s_{j}\right)=\left\{\begin{array}{cl}
s_{i} & \text { if } s_{i}=s_{j}, \\
0 & \text { if } s_{i} \neq s_{j} .
\end{array}\right.
$$


For every $r \in[0,1]$, play of $(r, r)$ is a pure strategy equilibrium.

The diagonal discontinuity means that the utilities sections are not sup norm precompact. The sections $\mathcal{U}_{i}$ are of the form $\left\{s_{i} \mapsto r \cdot 1_{\{r\}}\left(s_{i}\right): r \in[0,1]\right\}$. For every $\epsilon \in(0,1)$ there is a continuum (of size $1-\epsilon$ ) of functions in $\mathcal{U}_{i}$ at sup norm distance at least $\epsilon$ from each other.

Polynomials in the extensions of the utility sections are constant on a set of full Lebesgue measure in $S$ that can be expressed as a finite union of rectangles. Such functions cannot uniformly approximate the diagonally discontinous $u_{i}$.

The failure of integrability is also clear. For any $r>0$, joint play of any finitely additive $\nu_{i}$ satisfying $\nu_{i}\left(E_{i}\right) \in\{0,1\}$ and $\nu_{i}(r-1 / n, r) \equiv 1$ does not give a welldefined expected utility.

3.2.3. A diagonal discontinuity with no equilibria. The utilities $u_{i}\left(s_{i}, s_{j}\right)$ again fail to be continuous at $s_{i}=s_{j}$ in the following non-NCC game.

Example 3. The game $\Gamma_{3}$ is specified by $I=\{1,2\}, S_{i}=(0,1]$, and the symmetric utility functions,

$$
u_{i}\left(s_{i}, s_{j}\right)= \begin{cases}1 & \text { if } s_{i}<s_{j} \\ 0 & \text { if } s_{i} \geq s_{j}\end{cases}
$$

Again, the diagonal nature of the discontinuity makes the utility sections fail to be sup norm precompact so the game is not NCC. For any $\epsilon \in(0,1)$, the game fails to have an $\epsilon$-equilibrium.

3.2.4. An extensive form game. The following extensive form example is taken from Aumann's [2] treatment of the game theoretic problem of picking random functions on infinite sets.

Example 4. At time $t=1$, player 1 picks an action in $S_{1}=[0,1]$. Player 2 observes $s_{1}$ and at $t=2$, picks $a_{2} \in A_{2}=[0,1]$. The utility difference between $s_{1}=, \neq a_{2}$ is at least 1 .

Player 2's set of strategies, $S_{2}$, is a set of functions from [0,1] to [0,1]. If $S_{2}$ separates points in $S_{1}$, then for all $s_{1} \neq t_{1}$ and $s_{2} \neq t_{2}, d_{\mathcal{U}_{1}}\left(s_{1}, t_{1}\right) \geq 1$ and $d_{\mathcal{U}_{2}}\left(s_{2}, t_{2}\right) \geq 1$, so the game is not NCC.

The failure of measurability is quite striking. Aumann [1] showed that if $S_{2}$ is the set of Borel measurable functions, then there is no $\sigma$-field of subsets, $\mathcal{S}_{2}$, such that the product $\sigma$-field, $\mathcal{S}_{1} \otimes \mathcal{S}_{2}$ makes independent, countably additive randomization by the two players integrable. 


\section{Conclusions}

The intrinsic specification of a game involves only the pure strategies available to the players and the utilities. This paper has shown that the following five conditions are equivalent:

(1) the game can be regarded as a dense subgame of a compact and continuous game,

(2) the utilities are the uniform limits of polynomials in their extended sections,

(3) the game is integrable for all profiles of finitely additive strategies,

(4) the sections have compact sup norm closure, and

(5) the strategy sets are finitely approximable.

A game is nearly compact and continuous (NCC) if any of these conditions hold. For NCC games, there is a natural and minimal topological and measure theoretic structure, given by Fudenberg and Levine's 'most utility difference it can make to anyone' pseudo-metric $d_{\mathcal{U}_{i}}$ and the corresponding Borel $\sigma$-field. These structures lead to existence, upper hemicontinuity of the equilibrium correspondence, and continuity of the approximate equilibrium correspondence. The use of finer topological structures, as typically happens with finitely additive strategies, lead to the unnecessary complication of having many equivalent strategies.

After identifying strategically equivalent strategies, the minimal structure is that of a game with compact metric sets of strategies and jointly continuous utilities. In this sense, Fan [6] and Glicksberg [8] had found the 'right' structures and conditions for equilibrium existence for games with infinite normal forms. Rather than imposing these structures and conditions as part of the specification of a game, the work here identifies these structures and conditions in the intrinsic specification of the game.

Normal form games fail to be NCC when they have diagonal discontinuities. Special types of diagonal discontinuities were exploited by Dasgupta and Maskin [3] and Simon [12] to prove equilibrium existence. The tightness of the NCC condition can be seen in Example 3 in which: equilibria do not exist, which implies that upper hemicontinuity fails; approximate equilibria do not exist; and large finite approximations to the game entirely misrepresent the infinite game. The treatment of non-NCC games in [14] indicates that this misrepresentation can be interpreted as a failure of the usual model of the continuum. It also presents an alternative model of infinite sets that does not misrepresent the limits of finite approximations.

There is a second set of questions that flow from the answers to the first set. They are, "Do continuous compactifications, finite approximations, and finitely additive mixtures 'work' for games that are not NCC?" At one level, the answer is clearly 
not - continuous compact imbeddings exist if and only if the game is NCC. If $\Gamma$ is not NCC, then for at least one player $i$, there will exist no continuous $\hat{u}_{i}$ on $\widehat{S}$ that is equal to $u_{i}$ when restricted to $S$. Thus, the second set of questions reduces to whether or not continuity can be dispensed with in compact games.

For a game $\Gamma=\left(S_{i}, u_{i}\right)_{i \in I}$ with compact metric $S$, Simon and Zame [13] show that equilibria exist for the related games $\left(S_{i}, \hat{v}_{i}\right)_{i \in I}$. The utility functions $\hat{v}_{i}$ are measurable selections from the pointwise convex hull of the closure of the graph of the $u_{i}$ in $S \times \mathbb{R}^{I}$. The measurable selection approach is meant to dispense with continuity. As detailed in [14], the selection approach has two major weaknesses: the first is related to taking selections from the convex hull, or indeed, from the closure of the graph without convexification; the second has to do with the existence of profitable deviations. For non-NCC games, [14] shows that alternate, nonstandard models of infinite sets are much better For NCC games, the present work shows that the usual models are quite sufficient.

\section{REFERENCES}

[1] Aumann, R. Borel structures for function spaces. Illinois Journal of Mathematics (5), 614-630 (1961)

[2] Aumann, R. Mixed and behavior strategies in infinite extensive games. Annals of Mathematical Studies (52), 627-650 (1964).

[3] Dasgupta, P. and E. Maskin. The existence of equilibrium in discontinuous economic games, I: theory. Review of Economic Studies LII 1-26 (1986).

[4] Dugundji, J. Topology. Allyn and Bacon: Boston (1966).

[5] Dunford, N. and J. T. Schwartz. Linear Operators, Part I: General Theory. John Wiley \& Sons: New York, N.Y. (1957).

[6] Fan, K. Fixed point and minimax theorems in locally convex topological linear spaces. Proceedings of the National Academy of Science, U.S.A. 38, 121-126 (1952).

[7] Fudenberg, D. and D. Levine. Limit games and limit equilibria. Journal of Economic Theory 38, 261-279 (1983).

[8] Glicksberg, I. L. A further generalization of the Kakutani fixed point theorem, with application to Nash equilibrium points. Proceedings of the American Mathematical Society 3, $170-174$.

[9] Marinacci, M. Finitely additive cournot and Nash equilibria. Photocopy, Department of Economics, Northwestern University (1993).

[10] Marinacci, M. Finitely additive and epsilon Nash equilibria. International Journal of Game Theory 26, 3, 315-333 (1997).

[11] Nash, J. F. Equilibrium points in $n$-person games. Proceedings of the National Academy of Science, U.S.A. 36, 48-49 (1950).

Springer-Verlag.

[12] Simon, L. Games with discontinuous payoffs. Review of Economic Studies LIV, 569-597 (1987). 
[13] Simon, L. and W. Zame. Discontinuous games and endogenous sharing rules. Econometrica 58(4), 861-872 (1990).

[14] Stinchcombe, M. B. General normal form games. Working Paper, Department of Economics, University of Texas at Austin, May 2001.

King's College, Cambridge, CB2 1St, UK and Department of Economics, Department of Economics, University of Texas, Austin, TX 78712-1173 USA and Department of Economics, University of California, Los Angeles, CA 90024 USA 